\title{
Un tercer espacio crítico-reflexivo: sobre la identidad académica activista
}

A Critical-Reflective Third Space: On the Activist Academic Identity

Fecha de recepción: 30 DE MAYO DE 2017 / Fecha de aceptación: 25 DE NOVIEMBRE DE 2017 / Fecha de disponibilidad en línea: SEPTIEMBRE 2018

Encuentre este artículo en http://magisinvestigacioneducacion.javeriana.edu.co/

\section{Resumen}

Este artículo explora la construcción de una identidad académica activista como un tercer espacio crítico-reflexivo. Un tercer espacio resulta clave para reimaginar una identidad académica activista más allá de una relación asimétrica con lo subalterno. Así, políticas de afinidad y solidaridades situadas reorientan esta relación, que desdibuja la división entre el conocimiento teórico y el conocimiento empírico con y sobre los movimientos sociales. Este artículo reflexiona sobre la producción de una identidad académica activista como tercer espacio, a partir del estudio sobre el movimiento estudiantil chileno, y considera las potencialidades, límites y desafíos de esta identidad como práctica de transformación colectiva.

\section{Palabras clave}

Identidad; movimiento social; ciencias sociales; tercer espacio; activismo

\begin{abstract}
This article explores the construction of an activist academic identity as a critical-reflective third space. A third space is pivotal to re-devise an activist academic identity beyond an asymmetric relationship with the subaltern. This way, the situated affinity and solidarity policies will redirect such relationship, which fades the division between theoretical knowledge and empirical knowledge on and thanks to the social movements. This paper reflects on the emergence of an activist academic identity as a third space based on the study of the Chilean student movement. It also addresses the potentials, limitations and challenges to this identity as a collective transformation practice.
\end{abstract}

\section{Keywords:}

Identity; social movement; social sciences; third space; activism

Para citar este artículo / To cite this article

Hernández-Santibañez, I. (2018). Un tercer espacio crítico-reflexivo: sobre la identidad académica activista. magis, Revista Internacional de Investigación en Educación, 11 (22), 99-112. doi: 10.11144/Javeriana.m11-22.tecr 


\section{Introducción}

¿Cómo y qué caracteriza la construcción de una identidad académica-activista? ¿Qué desafíos subyacen a la construcción de una identidad académica activista desde la investigación sobre movimientos sociales en el Sur global, pero desde el Norte global? ¿Es posible repensar esta identidad como un borde fronterizo o como un lugar común? ¿Qué implicancias tiene esta relación en la construcción de conocimientos y en la dimensión política de la investigación con —y sobre - los movimientos sociales? Este artículo aborda estas preguntas desde el debate sobre la construcción de un tercer espacio (Routledge, 1966; Soja, 1999), imbricado a una identidad académica activista-reflexiva (Autonomous Geographies Collective, 2010; Hamm, 2015). A partir de esta reflexión teórica, oriento la discusión sobre la construcción de la identidad académica activista-reflexiva como un "compromiso ético" (Routledge, 1996) y a la vez crítico con respecto a la cultura política que configura la práctica investigativa con y sobre los movimientos sociales.

En primer lugar, abordo aquellos aspectos críticos que dan cuenta de los límites de una identidad académica activista que se construye desde una condición subalterna y de "privilegio epistémico" (Motta \& Nilsen, 2011 , p. 2). A partir del reconocimiento de estas limitaciones, planteo la necesidad de reimaginar esta identidad académica activista como la producción de un tercer espacio que, en opinión de Paul Routledge (1996, p. 400), constituye el lugar desde el cual es posible "negociar los sitios de la academia y el activismo". Esto es, negociarlos como espacios de acción política configurados no desde el reclamo de autenticidad, sino como espacios abiertos, relacionales y en permanente reconfiguración. La construcción de este tercer espacio se articula a solidaridades situadas (Nagar \& Geiger, 2007), y a través de las cuales, una identidad académica activista se sitúa como un nodo dialogante con los movimientos sociales, sin delimitar una relación opuesta entre el conocimiento teórico y el conocimiento empírico producido por los movimientos sociales.

A continuación, exploro la construcción de mi identidad académicaactivista reflexiva. Como tal, este proceso refiere a la construcción de un tercer espacio crítico-reflexivo imbricado a mi investigación doctoral sobre el Movimiento Pingüino de 2006 y las geografías de construcción política del movimiento estudiantil chileno (Hernández, 2013a, 2013b, 2017). La construcción de esta identidad académica activista no representa en sí un proceso de "implementación lineal" (Derickson \& Routledge, 2015). Por el contrario, la producción de esta identidad como tercer espacio se engarza, más bien, en un proceso de reflexión y construcción desde el cual me posiciono en relación con el campo de investigación y a las formas de construcción del mismo. Como tal, es un proceso mediado por emociones, compromisos políticos y solidaridad que, a su vez, se constituye como un borde fronterizo y como un lugar común en el que continuamente reviso la construcción de mi identidad académica activista como tercer espacio. Finalmente, reflexiono sobre la investigación académica activista con y sobre los movimientos sociales y de cuáles son los dilemas, desafíos y límites de un tercer espacio crítico reflexivo que, en un contexto academicista orientado a la producción de una subjetividad académica neoliberal, articula ambos, la investigación y la docencia como una acción política comprometida con un cambio y transformación social. 


\section{Sobre los dilemas y límites en la construcción de una identidad académica activista}

Desde las Ciencias Sociales, la discusión y el debate sobre la construcción de una identidad académica activista se suelen situar en la perspectiva de procesos de investigación políticos/colaborativos que configuran una identidad comprometida con el reclamo de mayor justicia social de parte de comunidades y sujetos históricamente marginados. Birke Otto y Philipp Terhorst (2011) reflexionan, a partir de sus propias experiencias de investigación con movimientos sociales en el Sur global, sobre los límites y las posibilidades de que una investigación académica activista pueda superar una condición subalterna estructural y, a la vez, volverse una praxis transformadora.

Un aspecto central en esta praxis refiere a la dimensión reflexiva, entendida "como un esfuerzo de quienes investigan por situarse a sí mismos en relación al campo de investigación" (Routledge \& Derickson, 2015, p. 1). En opinión de Richa Nagar y Susan Geiger (2007), esta dimensión se ve limitada al enfocarse en un nivel individual que no considera las condiciones estructurales y la comprensión de cómo estas delimitan esa dimensión reflexiva. Al respecto, se vuelve imperativo "debatir sobre las estructuras, así como también sobre los procesos económicos, políticos e institucionales que sustentan el contexto de la experiencia de trabajo de campo y de cómo estas influyen y determinan [esta dimensión de reflexividad]" (Nagar \& Geiger, 2007, p. 269).

Otto y Terhorst (2011) priorizan un análisis sobre la construcción de una identidad académica activista a partir de estas condiciones estructurales. Su análisis plantea que uno de los aspectos críticos de este proceso tiene relación con cómo estas condiciones estructurales articulan la construcción de esta identidad imbricada a una distinción geográfica entre Norte y Sur. En opinión de ambos autores, la posibilidad de articular la investigación académica activista a procesos de investigación colaborativos y participativos, en particular, desde la perspectiva de un investigador activista del Norte global cuya investigación es sobre y con los movimientos sociales en el Sur global, se halla estructuralmente determinada por esta distinción geográfica. Esta distinción, a su vez, reproduce una capacidad de agencia que es desigual con respecto "al poder en relación con la interconexión y movimiento" (Massey, 1993, p. 62) que configuran las distintas "geometrías de poder" (Massey, 1993) en una investigación colaborativa y participativa. En opinión de Doreen Massey (1993), estas geometrías de poder remiten a la constitución de un mapa en el que distintos grupos sociales, así como también individuos se estructuran a través de diferentes posiciones de poder. En un mapa de geometrías de poder, estos distintos actores se sitúan diferenciadamente con respecto "al movimiento e interconexión" (p. 62). Massey (1993) argumenta que "desde esta perspectiva, no solo interesa quién se mueve y quién no, aunque esto es en sí mismo un elemento importante; sin embargo, estas distintas posiciones se refieren al poder en relación con la interconexión y movimiento" (p. 62).

Desde un enfoque de investigación colaborativa estas geometrías de poder operan a través de "un entorno en el cual las diferencias materiales, sociales, políticas y de poder son el resultado de historias de colonialismo, desarrollo y realidades locales" (Sultana, 2007, p. 374). Es decir, las geometrías de poder configuran una identidad académica activista, anclada a una condición subalterna, como una "posible relación subyacente de explotación e interdependencia" (Otto \& Terhorst, 2011, p. 201). 
Los problemas y dilemas de esta relación subalterna en la investigación activista no son nuevos (Diversi \& Finley 2010; Frenzel \& Sullivan, 2009; Ghorashi \& Wels, 2009; Veissiere, 2010) y, no obstante, "rara vez son abordados y problematizados en el discurso académico" (Otto \& Terhorst, 2011, p. 201).

Un primer dilema reconoce la imposibilidad de traducir las experiencias producidas desde el Sur en el pensamiento dominante eurocéntrico. Birke Otto y Philipp Terhorst (2011) plantean que esta imposibilidad de traducción y representación del conocimiento se halla precisamente en la dificultad de quien investiga de liberarse de ciertos cánones académicos convencionales $y$, por consiguiente, de poder traducir esta experiencia en "un lenguaje que es reconocible y publicable" (p. 209).

Un segundo dilema deriva de la "imposibilidad de poder escapar de ciertos roles predeterminados" (Otto \& Terhorst, 2011, p. 209). Ahora bien, este rol académico activista inevitablemente se nutre de la posibilidad de utilizar ciertos privilegios que derivan de la movilidad de su posición (Terhorst, 2009). Sin embargo, ambos autores plantean que esta identidad y su relación de movilidad se configuran desde una condición desigual que de modo estructural emerge a partir de una relación subalterna. Por ejemplo, la posición de movilidad no se constituye como una condición común sino más bien se estructura como un poder de decisión que solo involucra a quien investiga. En esta relación desigual, las comunidades permanecen "fijas en sus lugares y estructuralmente excluidas de obtener ciertos privilegios" (Otto \& Terhorst, 2011, p. 210) derivados de esta relación de movilidad.

Birke Otto y Philipp Terhorst (2011) señalan además que la identidad académica activista corre el riesgo de hallarse atrapada en una dinámica de relación subalterna. A partir de esta relación subalterna, esta identidad expresa la necesidad de transformación (Žižek, 1997). Sin embargo, ello no implica la transformación de las dinámicas de acceso al poder y de movilidad, porque son precisamente estas condiciones las que sustentan la construcción de la identidad del investigador activista comprometido con los cambios sociales. Como consecuencia, esta identidad se articula a través de una posición subalterna "que mantiene su inmovilidad, reducida a la vez a una permanente dependencia desde los privilegios del investigador", perpetuando "una relación jerárquica entre quienes pueden dar y aquellos que solo pueden recibir" (Otto \& Terhorst, 2011, pp. 210-211).

Un tercer obstáculo obedece a la imposibilidad de construir nuevas relaciones toda vez que estas se ven limitadas por la incapacidad de los participantes de reconocer las relaciones de poder que ellos mismos reproducen (Otto \& Terhorst, 2011). Por cierto, esto último no niega que la construcción de una identidad académica activista se imbrique en una dimensión espacial, precisamente por la negociación implícita en la relación social que se establece entre el investigador activista y las comunidades que sufren la marginación y opresión del capitalismo. Sin embargo, esta imposibilidad hace referencia a una relación de dependencia y, a la vez, a una incapacidad de superar esta condición hegemónica subalterna.

La identificación de estos dilemas plantea la necesidad de abordar aquellos desafíos que se engarzan a la construcción de un proceso colaborativo de investigación activista. Sin embargo, desde el reconocimiento de las relaciones de poder hegemónicas, esta aproximación es marginal con respecto a la posibilidad de reimaginar alternativas sobre nuevas formas de construcción de una identidad académica activista. Esta limitante refiere a la dominación y hegemonía de la condición subalterna, como un aspecto nodal de la construcción de una identidad académica activista. A su vez, 
esta condición subalterna remite a una dimensión de totalidad, que niega a la vez la posibilidad de reimaginar la condición de lo subalterno como una dimensión de identidad que opera relacionalmente.

Desde un pensamiento binario, la construcción de una identidad académica es superpuesta a una distinción entre un Norte global y un Sur global, lo que supone a la vez conceptualizar esta relación como dentro de o fuera de (Massey, 1999). Por otra parte, un pensamiento relacional reconfigura a la vez la construcción de esta identidad como un proceso en el cual distintos elementos "se interconectan y son a la vez constitutivos de esta identidad" (p. 12). Es decir, como un significado de identidad en movimiento y que puede fluir, como identidad, en una dimensión relacional tanto dentro de sí como fuera de esta relación con lo subalterno. Cabe decir que cuando esta dimensión de movimiento respecto a lo subalterno es reconocida, es a la vez enmarcada en la imposibilidad de producir una voz propia precisamente porque esta voz "requiere de una expresión desde la racionalidad y el lenguaje del orden simbólico dominante" (Otto \& Terhorst, 2011, p. 205).

Por otra parte, una hegemonía total de la condición subalterna niega la posibilidad de ampliar la narrativa propia dentro de los movimientos sociales y de facilitar, por lo tanto, una investigación activista colaborativa articulada a una traducción recíproca entre narrativas y lenguajes que pueden ayudar a encontrar una manera más exitosa de ampliar las luchas sociales (Santos, 2013). Por ejemplo, el significado de exclusión que imbrica a los distintos dilemas de una identidad académica activista y a partir del cual se articula una relación colaborativa activista con los movimientos sociales, podría no necesariamente coincidir con la narrativa propia de los movimientos sociales.

Subyace a esta narrativa la convicción de que las diferentes luchas políticas e intelectuales dentro de los movimientos sociales no refieren a la necesidad de ser incluidos. Más bien, estas luchas se articulan contra el modo y las formas de vida que el capitalismo — como modelo económico, cultural y social- genera. Por consiguiente, no se constituye como una lucha circunscrita a una demanda de inclusión, sino más bien a una transformación radical, como una lucha política y cultural sobre los modos de vida en una sociedad capitalista.

El reconocimiento de una narrativa propia no solo permite visibilizar las luchas de los movimientos sociales como un proyecto que es a la vez político, cultural e intelectual, sino también propone que un dilema central en la investigación activista requiere desaprender antes de aprender (Santos, 2012b). Esta idea del desaprender sitúa a la investigación activista como un proceso abierto, relacional y siempre en proceso de construcción. Desde esta perspectiva, el principal reto de la investigación activista comprometida con combatir las injusticias sociales (Otto \& Terhorst, 2011) requiere al mismo tiempo reimaginar la construcción de una identidad académica activista espacialmente. Es decir, como un tercer espacio que a su vez posibilita ampliar los límites que subyacen al dominio intelectualteórico en el espacio de la academia, a través de una identidad académica activista, que intenta superar la dicotomía de incompatibilidad imbricada a una condición subalterna.

\section{La identidad académica activista como tercer espacio}

La construcción de un tercer espacio (Soja, 1999) representa un lugar común, un espacio de resistencia que articula la posibilidad de reimaginar de un modo distinto la identidad académica activista. Por medio de la 
producción de un tercer espacio, el investigador activista articula un rol y compromiso entre dos mundos que parecen incompatibles. Es decir, un tercer espacio como un borde fronterizo (Motta, 2013), en movimiento entre el mundo académico y el mundo del activismo y por consiguiente como un lugar desde el cual "se negocia la ubicación de [ambos]" (Routledge, 1996, p. 400).

En opinión de Paul Routledge (1996), ni la academia ni el activismo representan en sí espacios acabados, completos y por ende enmarcados en un significado de autenticidad. Ambos se constituyen en interrelación y como espacios en movimiento imbricados a la "inmediatez" (Bey, 1994). Como tal se construyen desde la experiencia vivida e inmediata. Por lo tanto, la producción de un tercer espacio y su negociación entre la academia y el activismo se sustentan desde el objetivo de "vivir la teoría a través de esta dimensión de lo inmediato" (Routledge, 1996, p. 401).

Por cierto, la naturaleza de la experiencia de inmediatez en la academia se enmarca en prácticas institucionales superpuestas a "legados de colonialismo" (Corbridge, 1993; Cumbers \& Routledge, 2004) y a "estructuras de poder y opresión, así como también a mitos culturales que las sustentan" (Juris \& Khasnabish, 2013, p. 371). No obstante esta naturaleza, la producción de un tercer espacio remite a un "intelectual específico" (Foucault, 1980), que articula este rol intelectual como un compromiso político por "combatir las distintas formas de poder que sustentan la actividad intelectual" (Routledge, 1996, p. 403). Sin embargo, una identidad académica activista como tercer espacio se sitúa, a diferencia del intelectual específico, más allá del mundo de la academia.

La producción de un tercer espacio simboliza un lugar donde la identidad académica activista "inicia un proceso de revisión" (Hooks, 1990, p. 145), que como tal es marginal y constituye un lugar de riesgos que a la vez demanda la existencia de "una comunidad de resistencia" (p. 149). Bell Hooks (1990) señala que este lugar de marginalidad no es una imposición sino una elección propia. Representa un espacio de resistencia que "nutre nuestra capacidad de resistir y que ofrece la posibilidad de crear e imaginar nuevos mundos y nuevas alternativas" (p. 152). A su vez, este espacio de resistencia se vuelve político en la medida en que subvierte la distancia y la práctica opuesta a una intelectualidad configurada como un espacio cerrado.

La producción de un tercer espacio se engarza con un "compromiso crítico" (Routledge, 1996) que articula su construcción en movimiento y como nexo entre la academia y el activismo. En este sentido, representa la posibilidad de un "intercambio crítico" (Hooks, 1990) que ambiciona "la construcción de nuevos puentes y de coaliciones políticas efectivas con todas las subjetividades radicales y resistencias colectivas" (Soja, 1999, p. 277). Como resultado, la academia deja de ser interpretada como una "institución monolítica y como un sitio estable para trabajar" (Routledge, 1996, p. 403).

La producción de un tercer espacio crítico-reflexivo "busca alterar aquellos espacios inestables de la academia y del activismo" (Routledge, 1996, p. 403), y aborda desde la dimensión reflexiva las políticas culturales imbricadas en su práctica, extendiendo a la vez el espacio ambiguo entre ambos (Hamm, 2015). Un aspecto central en la producción de este tercer espacio tiene relación con las prácticas que configuran el compromiso crítico de una identidad académica activista.

Tales prácticas refieren a la producción de "solidaridades situadas" (Nagar \& Geiger, 2007), como una praxis que se reconoce en una orientación epistemológica que vincula la dimensión crítica-reflexiva del investigador activista al reconocimiento de otras "formas de saberes" (Santos, 2012b). En este sentido, estas solidaridades se localizan en una "relación horizontal de aprendizaje mutuo" (Motta, 2011, p. 196), imbricadas a una orientación epistemológica del desaprender (Motta, 2011).

A este respecto, la producción de un tercer espacio crítico-reflexivo comporta la prefiguración de estas solidaridades situadas como una praxis imbricada en la experiencia diaria y a través de la cual la producción del conocimiento se problematiza política e intelectualmente como una lucha por la transformación del sentido común (Motta, 2011). De esta forma, la construcción de este tercer espacio se produce a través de "una afinidad específica de relaciones que tienen lugar temporalmente y que como tal hacen posible la concretización de estas prácticas" (Starodub, 2015, p. 166). ¿Qué implicancias supone esta dimensión temporal para la construcción y/o reimaginación de una identidad académica activista?

Una aproximación a esta interrogante implica reconocer que la producción de un tercer espacio crítico-reflexivo puede, a partir de la especificidad de relaciones sociales, configurarse y proyectarse a diferentes escalas. Es decir, la construcción de una identidad académica activista se configura desde lo espacial precisamente porque su construcción se imbrica a lo local/territorial, global o en intersección con ambas (Massey, 1992). Un argumento esencial en la discusión sobre la configuración de una identidad académica activista alude precisamente a un privilegio de movilidad que Otto y Terhorst (2011) plantean como una limitación estructural en la construcción de la misma y que podría argumentarse como inexorablemente determinada por una dimensión de temporalidad. Sin embargo, precisamente, esta expansión de lo espacial a diferentes escalas permite reconfigurar la construcción 
de una identidad académica activista como un proceso dinámico articulado a distintas temporalidades.

Esta dimensión temporal en la producción de un tercer espacio crítico-reflexivo introduce la idea de una identidad académica activista biodegradable (FlesherFominaya, 2010). Es decir, una identidad que se constituye desde una "contingencia temporal" (p. 400) y que se reconfigura continuamente desde lo espacial y a diferentes escalas. A partir de tal presupuesto, la limitante estructural de movilidad podría ser abordada epistemológicamente como la configuración de una identidad académica activista superpuesta a una multimovilidad, que como tercer espacio, se articula desde lo espacial y temporal, operando a través de diferentes escalas. Como tal conlleva, precisamente por esta relación de espacio-tiempo, la posibilidad, por una parte, de reimaginar la construcción de una identidad académica activista como un open-ended process y, por otra, de expandir las fronteras de una producción intelectual crítica-reflexiva más allá de una distinción binaria entre Norte y Sur.

\section{Identidad académica activista reflexiva: solidaridad y políticas de afinidad}

En junio de 2011, tuve la oportunidad de presentar parte de mi investigación doctoral en la Conferencia PILAS', organizada por la Universidad de Cambridge. Mi ponencia The Ongoing Pathways of the Right to Education in Chile: the Case of the Penguins' Revolution reflexionaba sobre la construcción política del movimiento estudiantil secundario de 2006, conocido como la revolución pingüina. Entre abril y junio de ese mismo año, cientos de miles de estudiantes secundarios, conocidos como pingüinos por el color negro y blanco de su uniforme escolar, se movilizaban en todo el país. A través de su protesta social, el movimiento pingüino logró instalar el debate político sobre el fracaso de un modelo educativo como mercado educacional (Carnoy, 2003), para demandar que la educación debía ser entendida como un derecho, no como un privilegio.

En mi presentación planteaba la necesidad de entender las claves políticas del movimiento pingüino de 2006 como inmanentes al surgimiento de un actor político que resultaba fundamental y esencial para la construcción y continuidad de la acción colectiva y la demanda política de educación gratuita y de calidad para todos, expresadas en las masivas movilizaciones estudiantiles del año 2011. ¿Por qué investigar el movimiento pingüino de 2006 ? y ¿en qué medida mi in-

1 Postgraduates in Latin American Studies Annual Conference "Legacies of the Past, Challenges of the Present: Inequality and Marginality in Latin America", University of Cambridge, 27 ${ }^{\text {th }}-29^{\text {th }}$ June 2011. vestigación sobre este movimiento estudiantil define la construcción de mi identidad académica activista? Una respuesta a estas interrogantes podría explicarse desde "la correlación entre identidad y participación en los movimientos sociales, en un contexto de acción colectiva" (Bobel, 2007, p. 148). Sin embargo, Chris Bobel (2007) cuestiona si esta transformación de identidad, a través de la participación en los movimientos sociales, refiere explícitamente al reconocimiento de una identidad como activista. Un segundo elemento tiene relación con la dimensión temporal imbricada en la movilidad del rol académico activista (Otto \& Terhorst, 2011) y con cómo esta dimensión de temporalidad impacta y/o limita la construcción de una identidad académica activista más allá de los tiempos y límites del trabajo de campo.

Kevin McDonald (2002) cuestiona lo que él define como "la nueva ortodoxia del paradigma de la identidad colectiva" (p. 109). Autores como Alberto Melucci $(1989,1996)$ plantean un significado de identidad colectiva como proceso y que toma en consideración una perspectiva constructivista de la acción colectiva. Por otra parte, McDonald (2002) enfatiza que más que la producción de identidad colectiva, lo que resulta clave para la acción colectiva de los movimientos sociales es la idea de solidaridad, entendida como la capacidad de invocación y de articulación transversal con otros actores sociales.

La noción de solidaridad se aproxima a la producción de "políticas de afinidad" (Routledge, 1996) que no necesariamente se enmarcan en el concepto de identidad sino en el de colectividad. Tampoco se limitan a una dimensión temporal, por ejemplo, a un período particular de movilización colectiva. Estas políticas de afinidad se constituyen desde la heterogeneidad, y por consiguiente desde lo espacial, articulándose a través de valores y creencias comunes, como una "estructura de sentimientos" (Williams, 1977) engarzadas a las experiencias e interpretaciones colectivas.

En la construcción de mi identidad académica activista, estas políticas de afinidad y solidaridad se conectan a la vez con mi propia experiencia de activismo político en el movimiento estudiantil universitario chileno que lideró las protestas estudiantiles en contra de la dictadura de Augusto Pinochet [11 de septiembre de 1973-11 de marzo de 1990] durante la década de los ochenta. En este sentido, hay una necesidad de expresar solidaridad, como un compromiso político por defender el derecho a la protesta que el movimiento pingüino expresa en su demanda por legitimar la movilización social en el Chile del período de postransición democrática. Ciertamente, este compromiso político, como política de afinidad, se sitúa como un tercer espacio que navega entre trayectorias y experiencias que no son temporalmente "alienables 
dentro de una historia única y particular" (Massey, 1999, p. 281). En este sentido, mi experiencia de activismo estudiantil puede ser reconocida como un espacio de solidaridad desde lo común y, desde una dimensión temporal, se sitúa como un "lenguaje de la experiencia vivida" (Vaneigem, 1983, p. 75). El reconocimiento de esta trayectoria de activismo estudiantil universitario resulta clave para justificar por qué investigar el movimiento estudiantil de 2006. Como tercer espacio, se imbrica a mi compromiso intelectual-crítico por entender cuáles son las nuevas formas de activismo estudiantil, democracia y participación política imbricadas en el movimiento pingüino de 2006.

Como un espacio crítico y de resistencia, reconozco las dinámicas de poder y conocimiento dentro de las ciencias sociales y de cómo un análisis empírico-teórico de estas nuevas formas de acción colectiva, particularmente desde las distintas teorías de los movimientos sociales, niega la posibilidad de reconocer otros saberes no academicistas en el análisis e interpretación sobre politicidad y nuevos actores sociales. En opinión de Boaventura de Sousa Santos (2012b), una idea de universalidad implícita en la producción del conocimiento sobre los movimientos sociales remite a una distinción en su construcción. Es decir, como un proceso posterior a las luchas sociales y, por consiguiente, como un saber academicista y un conocimiento o saber no academicista construido en las luchas sociales.

Al respecto, una tensión epistemológica dentro de la teoría de los movimientos sociales remite a equiparar esta idea de universalidad del conocimiento sobre movimientos sociales a categorías únicas, por ejemplo, estructurar su análisis a una condición de homogeneidad per se que no considera que la constitución de estos actores colectivos se manifiesta como "un proceso de construcción social que comprende heterogeneidad y una dimensión de fragilidad" (Melucci, 1989, p. 4). Esta distinción epistemológica es fundamental en la producción de un tercer espacio crítico-reflexivo que articula una política del conocimiento sobre movimientos sociales desde la heterogeneidad que los caracteriza y que, a la vez, enfoca la dimensión de lo contextual como un elemento primordial no solo en la construcción del conocimiento sobre los movimientos sociales, sino también en el reconocimiento de otros saberes no academicistas.

Ciertamente, el reconocimiento de otros saberes no academicistas que confluyen en las formas de interpretación empírico-teóricas sobre los movimientos sociales resulta fundamental para entender la construcción de mi propia identidad académica activista-reflexiva. Así, retomando la experiencia de mi presentación en la Conferencia PILAS de 2011, yo podría argumentar que la necesidad de subrayar el rol clave del movimiento pingüino de 2006 en la constitución y continuidad del movimiento estudiantil chileno se engarza al reconocimiento de un conocimiento construido en las luchas sociales y que como tal se imbrica a mi propia experiencia de activismo estudiantil.

Por lo tanto, esta experiencia de activismo político en el movimiento estudiantil universitario de finales de la década de los ochenta es el lugar político-intelectual desde el cual sitúo el reconocimiento de un conocimiento no academicista. Representa en sí mismo un espacio de solidaridad y afinidad vinculado a mi propia experiencia de lucha estudiantil que por consiguiente no me hace ajena a la interpretación sobre el cómo de la construcción del conocimiento en los movimientos sociales. Sin embargo, el reconocimiento de este saber producido en las luchas sociales no excluye la posibilidad de vincular este conocimiento a aquellos saberes que son producidos después de las luchas sociales. Es decir, una identidad 
académica activista que no se enmarca como un insider/outsider en el sentido absoluto con respecto a los distintos espacios y territorios de producción del conocimiento con y sobre los movimientos sociales.

\section{Sobre la producción de un tercer espacio: reflexiones desde la construcción del conocimiento sobre movimientos sociales}

Una interrogante central en la construcción de mi propia identidad académica activista tiene relación con cómo producir el conocimiento de mi investigación doctoral sobre espacio y política en el movimiento estudiantil chileno sin que ello implique a la vez reinscribir un conocimiento teórico que se desvincula de la producción del conocimiento empírico generado por este movimiento estudiantil. Un desafío similar refiere a la dimensión temporal del trabajo de campo y de cómo, una vez que este concluye, se corre el riesgo de delimitar este conocimiento a una dimensión abstracta, es decir, como un "dualismo entre práctica y teoría" (Motta, 2011, p. 186), alejada de las políticas materiales de cambio social (Nagar \& Geiger, 2007) y prácticas sociales que, como luchas políticas y cognitivas, sustentan los intereses de los movimientos sociales.

¿Cómo investigar un movimiento estudiantil en el Sur global sin imponer al mismo tiempo categorías preestablecidas y producidas en el Norte global? Por ejemplo, cómo explorar la emergencia de este actor colectivo sin extrapolar su análisis a la categoría de homogeneidad o a una dimensión política que en el estudio de los movimientos sociales limita su análisis a su capacidad de generar transformaciones en el sistema político (Crouch \& Pizzorno, 1978; Pizzorno, 1978), más que a analizar el impacto de los movimientos sociales en la sociedad civil (Melucci, 1989). Los interrogantes de este tipo guían el desarrollo de esta investigación como un estudio cualitativo exploratorio que emplea algunas estrategias del "método de teoría de base constructivista" (Charmaz, 2006) y que a la vez combina con una "teoría de base informada" (Thornberg, 2012):

[La teoría de base informada] refiere a un "proceso de investigación fundamentado en los datos, a través del uso de los métodos de la teoría de base mientras que a la vez el proceso de investigación se retroalimenta tanto de los marcos teóricos como de la literatura de investigación existentes" (p. 249).

El uso de "conceptos de sensibilización" (Blumer, 1969) en esta investigación, como "puntos de partida para desarrollar más que limitar nuestras ideas" (Charmaz, 2006, p. 17) me permite guiar el análisis cualitativo de la investigación, conectando ambos: el conocimiento teórico y empírico como un espacio intelectual crítico que interroga desde los datos la dimensión relacional entre ambos. Es decir, explorar cómo pueden el conocimiento empírico y también el teórico recíprocamente informar, facilitar y aprender el uno del otro.

Esta condición de movimiento, como "un espacio que permite la ruptura [de ambos] y en ambas direcciones" (Routledge, 1996, p. 402) se articula a una reflexión crítica sobre cuáles son los límites de una investigación unidisciplinaria. Como tal, es un proceso imbricado a la teoría de base constructivista y también a la teoría de base informada que permite que esta investigación navegue a "nuevos terrenos" (Charmaz, 2014), ampliándose al desarrollo de una investigación interdisciplinar. De este modo, la geografía radical conecta con ambas disciplinas: sociología de la 
educación y sociología de los movimientos sociales, para reconfigurar su relación en el análisis del espacio y la política en el movimiento pingüino y su influencia en las geografías de construcción política del movimiento estudiantil chileno.

Un desafío en esta investigación tiene relación con cómo situar mi identidad sin pretender, por ejemplo, identificar la misma al rol del investigador como experto o remitirla a la presuposición ingenua de que el investigador llega al trabajo de campo sin un conocimiento previo. La investigación sobre movimientos sociales privilegia la idea de eliminar el sesgo existente tanto en la investigación cualitativa como participativa (Haug \& Teune, 2008) y limitar la relación entre el investigador y su trabajo de campo, por ejemplo, al uso de notas a pie de página (Haunss, 2004). En opinión de Marion Hamm (2015), siempre es necesario utilizar estrategias que reduzcan la ambigüedad, al distinguir explícitamente la identidad académica activista como posiciones diferenciadas. Es decir, aproximarse al trabajo de campo desde un rol de investigador que a priori podría garantizar que el rol académico activista alcance un cierto tipo de "distancia crítica" (Harvey, 1996).

Mi aproximación al movimiento estudiantil se articuló precisamente a través de la identificación de mi rol como estudiante de posgrado, al investigar las geografías de construcción política del movimiento estudiantil chileno. Sin embargo, esta estrategia no redujo las posibilidades de desarrollar políticas de afinidad. Esta posibilidad se inscribe en el contexto de las masivas movilizaciones estudiantiles que tuvieron lugar entre abril y noviembre de 2011. Durante el transcurso de mi trabajo de campo, mi aproximación a los activistas, y a los estudiantes movilizados se da precisamente en el contexto de las distintas movilizaciones, por ejemplo, marchas que fueron convocadas por el movimiento estudiantil. Otros espacios refieren a las distintas asambleas y foros de discusión que el movimiento estudiantil organizaba durante las ocupaciones de facultades y escuelas secundarias.

La posibilidad de producir relaciones de afinidad desde el reconocimiento de mi identidad como investigadora dependió justamente del grado de apertura de estos distintos espacios. Es decir, mi identidad y mi posición dentro del movimiento estudiantil se articulan de un modo horizontal, precisamente porque la condición de apertura de estos espacios se imbrica con la idea de vincular la demanda por una educación gratuita y de calidad para todos a un proceso de democratización masiva de la discusión política de esta demanda con distintos sectores de la sociedad.

Desde una dimensión temporal, ¿cómo la construcción del conocimiento como un tercer espacio crítico-reflexivo se articula en un escenario posterior al trabajo de campo? Como tercer espacio, se halla "siempre abierto a la posibilidad de reinscribirse en otros [espacios] que intenta transformar" (Routledge, 1996, p. 414). En este sentido, una dimensión de temporalidad no limita a su vez la condición de fluidez y movimiento de una identidad académica activista. Esta condición insta, por ejemplo, a reimaginar la academia como un espacio de resistencia y como un espacio político desde el cual es posible articular "diferentes tipos de accion[es] política[s]" (Routledge, 1996, p. 402), como mecanismos disruptivos de las relaciones de poder/conocimiento superpuestas en el mundo de la academia.

El desarrollo de talleres, grupos de lecturas sobre educación y neoliberalismo con estudiantes de posgrado, y también foros de discusión con estudiantes y activistas del movimiento estudiantil remiten a ciertas prácticas, a través de las cuales me propuse abordar las barreras espaciales entre 
el mundo académico y el activismo. Se constituye a la vez como un espacio performativo, a través de prácticas que representan formas de resistencia y de ocupación, tanto material como simbólica del espacio académico. Por ejemplo, el desarrollo de foros con estudiantes y activistas del movimiento estudiantil chileno permite ampliar las voces de las comunidades y movimientos sociales que históricamente han sido excluidos de este espacio intelectual. En este sentido, representa una estrategia que busca precisamente "dispersar el poder" (Zibechi, 2010) en y fuera del mundo de la academia, como "una forma de conexión, cooperación y comunicación con el mundo del activismo" (Routledge \& Derickson, 2015, p. 7).

Boaventura de Sousa Santos (2012a) plantea la necesidad de reconocer los conocimientos producidos en las luchas sociales e incorporar estos conocimientos populares en un diálogo con otros conocimientos científicos. Al respecto, las distintas prácticas mencionadas anteriormente se oponen a la idea de enmarcar a la educación en una relación binaria de insider/ outsider. Es decir, la educación, como espacio, enmarcada y articulada a la distinción de un conocimiento academicista/no academicista. La incorporación de un conocimiento popular, articulado a las luchas políticas y cognitivas de los movimientos sociales en el espacio académico procura transformar esta dimensión binaria en una línea más bien difusa.

Esta línea difusa entre distintos conocimientos se articula, por ejemplo, a través del desarrollo de grupos de lectura sobre educación y neoliberalismo. Desde una perspectiva epistemológica, estas prácticas conectan con una dimensión política-intelectual, basada en un diálogo entre distintos conocimientos y que, a la vez, disputa la noción de la educación, en un contexto de políticas neoliberales, como una solución tecnocrática, neutral, no ideológica y basada en evidencia (Redondo, 2011). Por ejemplo, en estos espacios se incorpora una definición de la educación desde la política como un significado contrahegemónico que enarbolan las distintas luchas del movimiento estudiantil chileno. Como práctica imbricada a una reflexión crítica no intenta, sin embargo, solapar una dimensión política de la educación sobre una tecnocrática. Al contrario, este debate se plantea como un espacio de discusión y reflexión sobre el carácter político/ideológico que subyace a una solución tecnocrática en educación. Como un tercer espacio, este debate intenta desestabilizar una visión hegemónica de la educación, y al mismo tiempo ampliar las fronteras de un rol académico intelectual al reconocer que las políticas de investigación no solo determinan las formas de construcción del conocimiento, sino que también como prácticas constituyen en sí mismas una acción político-intelectual.
Como tercer espacio, estas prácticas se configuran como "políticas de articulación" (Routledge, 1996), es decir, como espacios convergentes comunes entre el mundo del activismo y de la academia, imbricadas a una dimensión espacial precisamente por la producción de relaciones de afinidad y "solidaridades situadas" (Nagar \& Geiger, 2007), por ejemplo, a través de la producción de redes. De este modo, estas políticas de articulación buscan trascender la dimensión de temporalidad superpuesta a los diferentes horizontes de tiempo de la investigación con y sobre los movimientos sociales y más allá de una distinción binaria entre Norte y Sur. Como tal, es posible reinscribir estas políticas de articulación a una cosmovisión de luchas políticas que admiten la igualdad desde la diferencia y que, desde el reconocimiento al derecho de ser diferentes (Santos, 2013), buscan ampliar estas luchas desde lo común. Es decir, desestabilizar el dominio tradicional del mundo intelectual, como privilegio académico, que a su vez intenta ampliar y transformar el significado y dominio de lo público en el espacio de la academia.

\section{Discusión}

Un objetivo de este artículo ha sido reflexionar sobre la construcción de una identidad académica activista más allá de una relación binaria entre Norte/Sur, adscrita a un rol de movilidad y de condición subalterna en la investigación activista con y sobre los movimientos sociales. Su construcción como un tercer espacio crítico y reflexivo aborda estas limitantes desde dentro de un espacio académico, y a través de la producción de políticas de afinidad, prácticas solidarias y políticas de articulación. A su vez, la construcción de este tercer espacio da cuenta de la posibilidad de superar la distinción entre el mundo académico-intelectual y el mundo social, porque se imbrica a una estrategia por reposicionar el rol de la universidad como un espacio de lucha y resistencia. Esto último se conecta con la construcción de una identidad académica activista que reconoce su dimensión política en lo popular. Es decir, como un nodo teórico-empírico dialogante que construye un conocimiento intelectual-popular desde un aprendizaje horizontal, mutuo y colaborativo.

Una característica fundamental de un tercer espacio es que desdibuja precisamente las fronteras del mundo académico orientado a una producción de academicismo capitalista neoliberal. En este sentido, una interrogante abierta tiene relación con cuáles son los desafíos y los posibles límites en la producción de nuevos territorios de debate y análisis de esta identidad.

Un aspecto crítico en la construcción de una identidad académica activista y su articulación con los movimientos sociales tiene relación con la imposibilidad 
de escapar a ciertas relaciones de poder. La producción de solidaridades situadas se aproxima a estas dinámicas de poder sin que ello implique, sin embargo, negar que en una relación académica-activista colaborativa se superponen negociaciones de poder desiguales. En este sentido, el desafío refiere a cómo abordarlas. Para ello, un elemento central remite a la producción de una praxis prefigurativa horizontal y dialogante que intenta desestabilizar el rol tradicional de producción del conocimiento en la academia alejado de la producción de conocimientos y prácticas populares.

Esto último plantea, por consiguiente, nuevas interrogantes respecto a la investigación interdisciplinaria sobre movimientos sociales. Por ejemplo, una agenda de investigación sobre movimientos sociales, caracterizada por un mayor desarrollo interdisciplinar e internacionalización, esboza nuevos desafíos ante la posibilidad de que la construcción del conocimiento sobre movimientos sociales, articulada desde una praxis prefigurativa horizontal, supere los límites y barreras ya existentes en la investigación interdisciplinar sobre movimientos sociales.

Precisamente, el rol de una identidad académica activista es situarse en nuevas arenas y cuestionar estas contradicciones desde lo colectivo; es decir, como prácticas solidarias, localizadas en un contexto político más amplio, reimaginando a la vez nuevos territorios de lucha como líneas difusas entre el inside y outside. Es decir, una identidad académica activista en movimiento permanente entre una dimensión solidaria y la construcción de un significado de colectividad, que no se localiza en un sentido absoluto entre el centro y la periferia y por consiguiente más allá de la dicotomía Norte-Sur.

Esta dimensión de lo colectivo no solo disputa el lugar de la academia como un espacio exclusivo en la producción del conocimiento y por ende, su distancia con el mundo del activismo; sino que también abre nuevas interrogantes en relación con las posibilidades, desafíos y límites de una identidad académica activista que procura disputar y transformar, desde lo colectivo, la misión social de la universidad pública en tiempos de una mayor globalización neoliberal de las universidades y del conocimiento.

\section{Sobre la autora}

Ivette Hernández-Santibañez es PhD en Sociología en el UCL Institute of Education, University College London. Sus intereses de investigación se ubican en la intersección de las políticas económicas neoliberales en educación urbana y movimientos sociales, con un enfoque en políticas públicas progresivas de educación gratuita, movimientos estudiantiles de base y políticas democráticas radicales en América Latina.

\section{Referencias}

Autonomous Geographies Collective (2010). Beyond Scholar Activism: Making Strategic Practices Inside and Outside the Neoliberal University. ACME: An International E-Journal for Critical Geographies, 9 (2), 245275. Disponible en: https://www.acme-journal.org/index.php/acme/ article/view/868

Bey, H. (1994). Immediatism. Essays by Hakim Bey. Edinburgh: AK Press.

Blumer, H. (1969). Symbolic Interactionism: Perspective and Method. Englewood Cliffs, New Jersey: Prentice Hall.

Bobel, C. (2007). I'm Not an Activist, though I've done a Lot of It: Doing Activism, Being Activist and the 'Perfect Standard' in a Contemporary Movement. Social Movement Studies, 6 (2), 147-159. 
Carnoy, M. (2003). Las políticas educacionales de Chile desde una perspectiva internacional. En C. Cox (ed.). Políticas educacionales en el cambio de siglo. La reforma del sistema escolar de Chile, 115123. Santiago de Chile: Editorial Universitaria.

Charmaz, K. (2006). Constructing Grounded Theory. A Practical Guide through Qualitative Analysis. London: Sage Publications Ltd.

Charmaz, K. (2014). Constructing Grounded Theory. London: Sage.

Corbridge, S. (1993). Marxisms, Modernities and Moralities: Development Praxis and the Claims of Distant Strangers. Environmental and Planning D: Society and Space, 11 (4), 449-472. https:// doi.org/10.1068/d110449

Crouch, C. \& Pizzorno, A. (1978). The Resurgence of Class Conflict in Western Europe since 1968. London: Macmillan.

Cumbers, A. \& Routledge, P. (2004). Alternative Geographical Imaginations: Introduction. Antipode, 36 (5), 818-828. https://doi.org/10.1111/j.14678330.2004.00456.x

Derickson, K. \& Routledge, P. (2015). Resourcing Scholar-Activism: Collaboration, Transformation, and the Production of Knowledge. The Professional Geographer, 67 (1), 1-7. https://doi.org/10.1080 /00330124.2014.883958

Diversi, M. \& Finley, S. (2010). Poverty Pimps in the Academy: A Dialogue about Subjectivity, Reflexivity, and Power in Decolonising Production of Knowledge. Cultural Studies Critical Methodologies, 10 (1), 14-17.

Flesher-Fominaya, C. (2010). Collective Identity in Social Movements: Central Concepts and Debates. Sociology Compass, 4 (6), 393-404. https://doi. org/10.1111/j.1751-9020.2010.00287.x

Foucault, M. (1980). Power/Knowledge: Selected Interviews and Other Writings, 1972-1977. New York: Pantheon Books.

Frenzel, F. \& Sullivan, S. (2009). Globalisation from Below? ICTs and Democratic Development in the Project "Indymedia Africa". En O. F. Mudhai, W. J. Tettey \& F. Banda (eds.). African Media and the Digital Public Sphere, 165-182. Basingstoke: Palgrave Macmillan.

Ghorashi, H. \& Wels, H. (2009). Beyond Complicity: A Plea for Engaged Ethnography. En S. Ybema, D. Yanow, H. Wels \& F. Kamsteeg (eds.). Organisational Ethnography. Studying the Complexities of Everyday Life, 231-251. London: Sage.

Hamm, M. (2015). Reflexive Activist Scholarship between the Fields of Activism and Academia: Ethnographic Reflections on the Cultural Politics of Knowledge Production in Social Movement Research. En 20 ${ }^{\text {th }}$ International Conference on
Alternative Futures and Popular Protests. Manchester, Reino Unido: Manchester Metropolitan University.

Harvey, D. (1996). Justice, Nature and the Geography of Difference. Cambridge: Blackwell Publishers.

Haug, C. \& Teune, S. (2008). Identifying Deliberation in Social Movement Assemblies: Challenges of Comparative Participant Observation. Journal of Public Deliberation, 4 (1), 1-35. Disponible en: http://www.publicdeliberation.net/jpd/vol4/ iss $1 /$ art8

Haunss, S. (2004). Identität in Bewegung. Prozesse kollektiver Identität bei den Autonomen und in der Schwulenbewegung. Wiesbaden: VS Verlag für Sozialwissenschaften. Disponible en: https://shaunss.ipgovernance.eu/wp-content/ uploads/2011/10/haunss-2004-identitaet-inbewegung.pdf

Hernández, I. (2013a). Interview with Francisco Figueroa: Continuing the Conversation on the Chilean Student Movement. Journal of Social Science Education, 12 (3), 60-67. DOI: 10.4119/UNIBI/ jsse-v12-i3-1235. Disponible en: http://www. jsse.org/index.php/jsse/article/view/1235/1216

Hernández, I. (2013b). Which Education for Which Democracy: The Case of the Penguins' Revolution in Chile. En S. C. Motta \& M. Cole (eds.). Education and Social Change in Latin America, 185202. New York: Palgrave Macmillan.

Hernández, I. (2017). Space and Politics in the Penguins' Movement: Geographies of the Political Construction of the Chilean Student Movement (tesis doctoral). Institute of Education, University College London, Reino Unido. Disponible en: http://discovery.ucl.ac.uk/1549607/

Hooks, B. (1990). Yearning: Race, Gender and Cultural Politics. Boston: South End Press.

Juris, J. \& Khasnabish, A. (2013). Introduction: Ethnography and Activism within Networked Spaces and Transnational Encounters. En J. Juris \& A. Khasnabish (eds.). Insurgent Encounters: Transnational Activism, Ethnography, and the Political, 1-36. London: Duke University.

Massey, D. (1992). Politics and Space/Time. New Left Review, 196, 65-84.

Massey, D. (1993). Power-Geometry and a Progressive Sense of Place". En J. Bird, B. Curtis, T. Putnam, G. Robertson \& L. Tickner (eds.). Mapping the Futures. Local Cultures, Global Change, 60-70. London: Routledge.

Massey, D. (1999). Spaces of Politics. En D. Massey, J. Allen \& P. Sarre (eds.). Human Geography Today, 279-294. Cambridge: Polity Press.

McDonald, K. (2002). From Solidarity to Fluidarity: Social Movements beyond 'Collective Identity'. 
The Case of Globalisation Conflicts. Social Movement Studies, 1 (2), 109-128.

Melucci, A. (1989). Nomads of the Present: Social Movements and Individual Needs in Contemporary Society. London: Hutchinson Radius.

Melucci, A. (1996). Challenging Codes, Collective Action in the Information Age. New York: Press Syndicate of the University of Cambridge. Disponible en: http://voidnetwork.gr/wp-content/ uploads/2016/09/Challenging-Codes-CollectiveCollective-action-in-the-information-age-byAlberto-Melucci.pdf

Motta, S. C. (2011). Notes Towards Prefigurative Epistemologies. En S. C. Motta \& A. G. Nilsen (eds.). Social Movements in the Global South: Dispossession, Development and Resistance, 178-199. New York: Palgrave Macmillan.

Motta, S. C. (2013). The Storytellers of Critique: Becoming Otherwise in Practice and Theory, paper presented as the Counter-Conducts Workshop, University of Sussex, Reino Unido.

Motta, S. C. \& Nilsen, A. G. (2011). Social Movements and/in the Postcolonial: Dispossession, Development and Resistance in the Global South. En S. C. Motta \& A. G. Nilsen (eds.). Social Movements in the Global South: Dispossession, Development and Resistance, 1-31. New York: Palgrave Macmillan.

Nagar, R. \& Geiger, S. (2007). Reflexivity and Positionality in Feminist Fieldwork Revisited. En A. Tickell, E. Sheppard, J. Peck \& T. Barnes (eds.). Politics and Practice in Economic Geography, 267-278. London: Sage.

Otto, B. \& Terhorst, P. (2011). Beyond Differences? Exploring Methodological Dilemmas of Activist Research in the Global South. En S. C. Motta \& A. G. Nilsen (eds.). Social Movements in the Global South: Dispossession, Development and Resistance, 200-223. New York: Palgrave Macmillan.

Pizzorno, A. (1978). Political Exchange and Collective Identity in Industrial Conflict. En C. Crouch \& A. Pizzorno (eds.). The Resurgence of Class Conflict in Western Europe since 1968, 277-298. London: Macmillan.

Redondo, J. (2011). Mal Educados! El problema de la educación en Chile. Disponible en: http://vimeo. com/27377040

Routledge, P. (1996). The Third Space as Critical Engagement. Antipode, 28 (4), 399-419. https://doi. org/10.1111/j.1467-8330.1996.tb00533.x. Disponible en: https://onlinelibrary.wiley.com/doi/epdf/ 10.1111/j.1467-8330.1996.tb00533.x
Routledge, P. \& Derickson, K. (2015). Situated Solidarities and the Practice of Scholar Activism. Environment and Planning D: Society and Space, 33 (3), 391 407. https://doi.org/10.1177/0263775815594308

Santos, B. de Sousa (2012a, mayo 7). Entrevista al profesor Boaventura do Santos. Disponible en: https:// www.youtube.com/watch? $v=K B 6 R b Y W f z k 0$

Santos, B. de Sousa (2012b, mayo 7). ¿Por qué las epistemologías del Sur? Disponible en: https://www. youtube.com/watch? $v=3 a 7$ peos $6 \mathrm{LP} 8$

Santos, B. de Sousa (2013, octubre 11). Descolonización epistemológica del Sur. Disponible en: https:// www.youtube.com/watch?v=hb1yUnf8TQU

Soja, E. (1999). Thirdspace: Expanding the Scope of the Geographical Imagination. En D. Massey, J. Allen \& P. Sarre (eds.). Human Geography Today, 261-278. Cambridge: Polity Press.

Starodub, A. (2015). Post-representational Epistemology in Practice: Processes of Relational Knowledge Creation in Autonomous Social Movements. Interface: A Journal for and about Social Movements, 7 (2), 161-191. Disponible en: http:// www.interfacejournal.net/wordpress/wp-content/uploads/2015/12/Issue-7-2-Starodub.pdf

Sultana, F. (2007). Reflexivity, Positionality and Participatory Ethics: Negotiating Fieldwork Dilemmas in International Research. AMCE: An International E-Journal for Critical Geographies, 6 (3), 374 385. Disponible en: https://www.acme-journal. org/index.php/acme/article/view/786

Terhorst, P. (2009). The Role of Social Movements in Developing Public Alternatives in Urban Water Services (tesis doctoral). Loughborough University, Leicestershire, Reino Unido. Disponible en: https://core.ac.uk/download/pdf/2745094.pdf https://dspace.lboro.ac.uk/2134/9220

Thornberg, R. (2012). Informed Grounded Theory. Scandinavian Journal of Educational Research, 56 (3), 243-259. https://doi.org/10.1080/00313 831.2011 .581686

Vaneigem, R. (1983). The Revolution of Everyday Life. London: Rebel Press.

Veissiere, S. (2010). Making a Living: The Gringo Ethnographer as Pimp of the Suffering in the Late Capitalist Night. Cultural Studies Critical Methodologies, 10 (1), 29-39. https://doi.org/10.1177/ 1532708609351152

Williams, R. (1977). Marxism and Literature. Oxford: Oxford University Press.

Zibechi, R. (2010). Dispersing Power: Social Movements as Anti-State Forces. Edinburgh: AK Press.

Žižek, S. (1997). Multiculturalism, or, the Cultural Logic of Multinational Capitalism. New Left Review, 225, 28-51. 